\title{
Síndrome de superposición de hepatitis autoinmune y colangitis biliar primaria asociada a trombosis de vena porta post COVID-19
}

\section{Overlap syndrome of autoimmune hepatitis and primary biliary cholangitis associated with portal vein thrombosis post COVID-19}

\author{
Thalía Isabel Zumaeta Rodríguez', \\ Luis Enrique Carrasco Lozano², \\ Silvia Patricia Zamudio Romero², \\ Luis Alberto Maya Pérez ${ }^{2}$
}

\begin{abstract}
Zumaeta-RodríguezT, Carrasco-Lozano L, Zamudio-Romero S, Maya-Pérez L. Síndrome de superposición de hepatitis autoinmune y colangitis biliar primaria asociada a trombosis de vena porta post COVID-19. Rev Soc Peru Med Interna. 2021;34(4): 186- 189. DOI: https://doi.org/10.36393/spmi.v34i4.636
\end{abstract}

\begin{abstract}
RESUMEN
Presentamos el caso de una mujer de 53 años con antecedentes de artritis reumatoide que, tras recuperarse de un cuadro de COVID-19grave, desarrolló hemorragia digestiva como manifestación de hipertensión portal e insuficiencia hepática progresiva. Basados en la serología y ecografía, se diagnosticó un síndrome overlap de hepatitis autoinmune y colangitis biliar primaria asociado a trombosis de vena porta. La respuesta a la corticoterapia y anticoagulación fue favorable.

El compromiso hepático, inducido por el virus, está descrito en la COVID-19. Sin embargo, pueden desarrollarse enfermedades autoinmunes y fenómenos tromboembólicos, teniendo el hígado como órgano diana.

Palabras claves: COVID-19, SARS-CoV-2, hepatitis autoinmune, colangitis biliar primaria, trombosis venosa, vena porta.
\end{abstract}

\section{ABSTRACT}

We present the case of a 53-year-old woman with a history of rheumatoid arthritis who, after recovering from a severe COVID-19, developed gastrointestinal bleeding as a manifestation of portal hypertension and progressive liver failure. Based on serology and ultrasound, an overlap syndrome of autoimmune hepatitis and primary biliary cholangitis associated with portal vein thrombosis were diagnosed. The response to steroids and anticoagulation was favorable. Hepatic involvement, induced by the virus, has been described in COVID-19. However, autoimmune diseases and thromboembolic phenomena can develop, having the liver as a target organ.

Keywords: COVID-19, SARS-CoV-2, autoimmune hepatitis, primary biliary cholangitis, venous thrombosis, portal vein.

I Médica residente de medicina interna. Facultad de medicina, Universidad Nacional Mayor de San Marcos. Departamento de Medicina Interna, Hospital Nacional Arzobispo Loayza, Lima, Perú.

2 Médico internista. Departamento de Medicina Interna, Hospital Nacional Arzobispo Loayza, Lima, Perú.

\section{INTRODUCCIÓN}

A nivel mundial, para el mes de setiembre del 2021, la COVID-19 presenta una distribución global, con más 232 millones de personas infectadas y 4,7 millones de fallecidos. El Perú es uno de los países más afectados por 
esta enfermedad, con la mortalidad más alta a nivel mundial, con más de 600 fallecidos por 100000 habitantes (1). Esta enfermedad es causada por el virus SARS-CoV-2, con un espectro clínico variable desde pacientes asintomáticos hasta insuficiencia respiratoria severa. Sin embargo, este virus no solo presenta compromiso respiratorio sino que también puede afectar a diversos órganos y sistemas como el cardiovascular, gastrointestinal, renal, neurológico, entre otros, así como presentar complicaciones tromboembólicas, pudiendo llevar a una disfunción orgánica múltiple y muerte por compromisos no respiratorios. ${ }^{2}$ Además, se han descrito manifestaciones inmunológicas y formación de auto anticuerpos en pacientes que previamente no presentaban este tipo de patologías. ${ }^{3}$

Presentamos el caso de una paciente mujer de 53 años con antecedente de artritis reumatoide sin compromiso hepático previo que, tras superar un cuadro severo de infección por SARS-CoV-2, reingresó por manifestaciones de hipertensión portal e insuficiencia hepática.

\section{PRESENTACIÓN DEL CASO}

Mujer de 53 años con antecedente de artritis reumatoide diagnosticada dos años antes del ingreso, controlada, y en tratamiento con prednisona $5 \mathrm{mg}$ diario. Tuvo COVID-19 grave con insuficiencia respiratoria dos meses antes del ingreso, diagnóstico que fue confirmado con prueba molecular de reacción en cadena de polimerasa positiva. Negó antecedentes de alcoholismo y uso de fármacos hepatotóxicos como metotrexate.

La paciente reingresó por el servicio de emergencia con un tiempo de enfermedad de 15 días caracterizado por náuseas y vómitos a lo que se agregó dificultad respiratoria y sangrado digestivo. Al ingreso presentó presión arterial de $80 / 50 \mathrm{~mm} \mathrm{Hg}$, frecuencia cardiaca de 102 latidos por minutos, frecuencia respiratoria de 20 respiraciones por minuto y saturación de oxígeno de $97 \%$. Al examen físico se evidenció palidez severa, ictericia de piel y escleras, llenado capilar lento y tacto rectal positivo a melena. En los análisis de ingreso, presentó hemoglobina $6,6 \mathrm{~g} / \mathrm{dl}$, leucocitos $7180 / \mathrm{mm}^{3}$, plaquetas $283000 / \mathrm{mm}^{3}$, urea 15 $\mathrm{mg} / \mathrm{dl}$, creatinina $0,45 \mathrm{mg} / \mathrm{dl}$, transaminasa glutámico oxalacética (TGO) $133 \mathrm{U} / \mathrm{L}$, transaminasa glutámico pirúvica $17 \mathrm{U} / \mathrm{L}$, fosfatasa alcalina (FA) $444 \mathrm{U} / \mathrm{L}$, bilirrubinas totales $7,76 \mathrm{mg} / \mathrm{dl}$, bilirrubina directa $7,07 \mathrm{mg} /$ dl, proteínas totales 4,28 mg/dl, albúmina 1,62 mg/dl y tiempo de protrombina 27,5 segundos con INR 2,13. En la ecografía abdominal se concluyó en hepatopatía crónica difusa asociada a líquido libre perihepático. Recibió soporte hemodinámico y transfusional hasta ser estabilizada y se le realizó una endoscopia digestiva alta donde se evidenció várices esofágicas (VVEE) grado III con signos rojos, realizándosele la primera sesión de endoligadura (Figura 1). Fue admitida a hospitalización de medicina interna para continuar el manejo.

Durante la hospitalización completó el tratamiento con octreotide por cinco días sin evidenciarse nuevo episodio de sangrado digestivo. Se revisó la historia clínica de

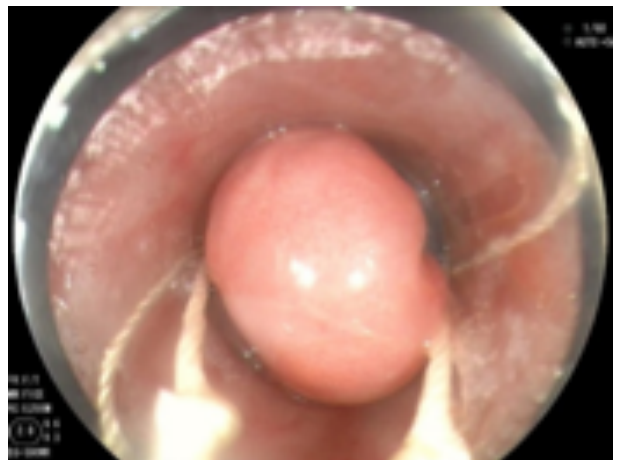

Figura I. Endoligadura de várice esofágica

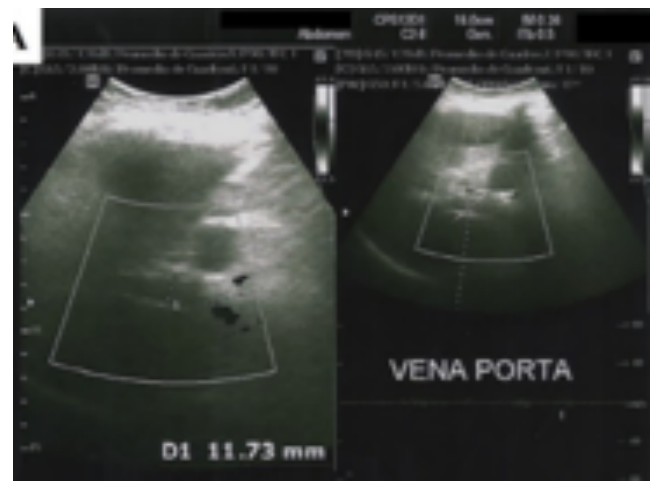

Figura 2. Ecografía Doppler de vena porta con ausencia de flujo, sugestivo de trombosis portal.

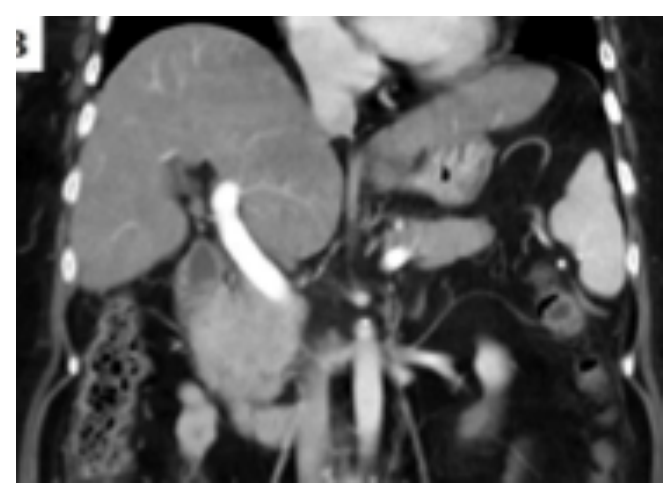

Figura 3. Reconstrucción coronal de angiotomografía contrastada de la vena porta realizada un mes después del inicio de la anticoagulación, con visualización homogénea del contraste en su interior, sin evidencia de trombosis.

la hospitalización previa por COVID-19, donde no se describió signos clínicos de hepatopatía crónica ni de hipertensión portal pero en los análisis se encontró un perfil hepático con TGO 138 U/L, TGP 58 U/L, FA 499 $\mathrm{U} / \mathrm{L}$, proteínas totales $6,10 \mathrm{mg} / \mathrm{dl}$, albúmina $2,74 \mathrm{mg} / \mathrm{dl}$ y tiempo de protrombina 21,8 segundos con INR 1,85. Estos hallazgos laboratoriales eran sugestivos de insuficiencia hepática con daño hepatocelular y colestásico. Debido al desarrollo de hipertensión portal severa que condicionó VVEE grado 3, se planteó la posibilidad de una trombosis portal. Se realizó una ecografía Doppler portal hallándose 
Tabla I. Evolución del perfil hepático de la paciente.

\begin{tabular}{|c|c|c|c|c|c|c|c|}
\hline Día de hospitalización & -60 & I & 12 & 28 & 35 & 39 & 51 \\
\hline Bilirrubinas totales, $\mathrm{mg} / \mathrm{dl}$ & & 7,76 & 13,49 & & $9,7 \mathrm{I}$ & 8,05 & \\
\hline Bilirrubina directa, mg/dl & & 7,07 & 13,40 & & 9,11 & 7,80 & \\
\hline TGO, U/L & 138 & 133 & 311 & 176 & 256 & 245 & 176 \\
\hline TGP, U/L & 58 & 17 & 78 & 45 & 81 & 98 & 63 \\
\hline $\mathrm{FA}, \mathrm{U} / \mathrm{L}$ & 499 & 444 & 815 & 758 & 860 & 679 & 542 \\
\hline GGT, U/L & & & & & 3290 & 2884 & 2136 \\
\hline Proteínas totales, mg/dl & 6,10 & 4,28 & 5,47 & 5,71 & 5,46 & 4,92 & 5,29 \\
\hline Albúmina, mg/dl & 2,74 & 1,62 & 2,50 & 2,56 & 2,69 & 2,36 & 2,52 \\
\hline Globulinas, mg/dl & 3,36 & 2,66 & 2,97 & 3,15 & 2,74 & 2,56 & 2,77 \\
\hline Tiempo de protrombina, seg. & 21,8 & 27,5 & 11,2 & 16,9 & 14,6 & & 14,1 \\
\hline INR & 1,85 & 2,13 & 0,95 & $\mathrm{I}, 25$ & $\mathrm{I}, 08$ & & $\mathrm{I}, 04$ \\
\hline
\end{tabular}

un hígado de $150 \mathrm{~mm}$, de bordes uniformes y ángulos romos, parénquima heterogéneo con presencia de líquido libre perihepático, vías biliares intrahepáticas no dilatadas, vena porta proximal de $11 \mathrm{~mm}$ de calibre con ausencia del flujo al estudio de Doppler color y espectral, concluyéndose en signos ecográficos de trombosis portal (Figura 2). Se inició anticoagulación terapéutica con enoxaparina con enoxaparina a dosis de $60 \mathrm{mg}$ SC cada 12 horas y se retomó la corticoterapia a dosis bajas una vez reiniciada la vía oral. Enla evolución, la ictericia de piel y escleras se intensificaron, realizándose controles seriados de perfil hepático (Tabla 1). Se evidenció incremento de las transaminasas y un marcado patrón colestásico. Los estudios de hepatitis virales fueron negativos. Por el antecedente de artritis reumatoide y el comportamiento clínico-laboratorial, se solicitó estudios de autoinmunidad: anticuerpos antinucleares (ANA) 3+ con patrón citoplasmático, anti-DNA negativo, anticuerpos anticitoplasma de neutróficos (ANCA) C y $\mathrm{P}$ negativos, antimúsculo liso (ASMA) negativo y antimitocontriales (AMA) 1/200 con serotipo M2, anticardiolipinas IgG e IgM negativos, inmunoglobulina $G$ cualitativo positivo. Además, se realizó un estudio de elastografía hepática evidenciando una rigidez de 9,6 kPA, clasificándose como F2 para colestasis según la escala METAVIR.

Al aplicar los criterios diagnósticos de hepatitis autoinmune (HAI), la paciente presentó un valor de 13 puntos clasificándose como HAI probable. Por el perfil hepático marcadamente colestásico con valor de gamma glutamil transferasa (GGT) superior a $3000 \mathrm{U} / \mathrm{L}$ (VN menor a $40 \mathrm{U} / \mathrm{L}$ ) también se sospechó una colangitis biliar primaria (CBP). Por lo tanto, se planteó un síndrome de superposición, overlap, entre HAI y CBP asociado a artritis reumatoide $y$ trombosis portal en el contexto de una paciente en estado post COVID-19, como causas de la falla hepática e hipertensión portal severa. Se decidió incrementar la dosis de prednisona a $1 \mathrm{mg} / \mathrm{kg}$ y agregar ácido ursodesoxicólico (AUDC). Tras dos semanas de tratamiento con mejoría clínica y laboratorial, se redujo progresivamente la dosis de prednisona y se agregó azatioprina como ahorrador de corticoides. En la angiotomografía de la vena porta realizado tras un mes de anticoagulación, se observó un calibre de dicha vena de $11 \mathrm{~mm}$, sin evidencia de trombosis (Figura 3). Se evidenció mejoría clínica de la ictericia y del perfil hepático con disminución de las bilirrubinas y de la actividad de las enzimas hepáticas (Tabla 1). La paciente fue dada de alta con prednisona, azatioprina, AUDC y anticoagulación con warfarina y controles de INR en rango terapéutico.

\section{DISCUSIÓN}

La HAI y CBP son trastornos inmunológicos con compromiso primario hepático con características clínicas, bioquímicas, serológicas e histológicas propias. La HAI se caracteriza por presentar autoreactividad del hepatocito con daño hepatocelular, mientras que en la CBP el daño se da a nivel de los canales biliares con compromiso colestásico (4). Durante muchos años se han considerado entidades completamente separadas con mecanismos fisiopatológicos separados, pero hoy en día se conoce que se puede presentar concomitantemente llamándose síndrome de sobreposición u overlap. Hasta el momento es un desafío realizar el diagnóstico de un síndrome de sobreposición porque no existe ningún marcador inmunológico específico que permita hacerlo con exactitud. ${ }^{5}$

En nuestra paciente, inicialmente se consideró el diagnóstico de HAI probable en base a los criterios diagnósticos originales del Grupo Internacional de Hepatitis Autoinmune, al presentar un valor de 13 puntos. Es importante mencionar que además la paciente presentaba un cuadro severo de HAI con ictericia y coagulopatía definida por un valor de INR mayor a 1,5 ; y, se ha descrito que en estos casos la sensibilidad de los criterios anteriormente indicados decae y es necesario el inicio de corticoterapia de forma urgente. ${ }^{6}$ Como se ha mencionado, el patrón de daño característico de HAI es hepatocelular con incremento de la actividad de las transaminasas séricas, pero nos encontramos frente a un patrón de daño predominantemente colestásico, por los valores de FA y GGT elevados, especialmente este último que se encontraba más de 80 veces por encima del valor superior normal. Es este contexto se debe plantear la 
posibilidad de un síndrome overlap entre HAI y CBP. No existen criterios completamente válidos para plantear este diagnóstico y se suele requerir la biopsia hepática, la cual puede ser difícil de obtener en muchos casos, por la presencia de ascitis o trastornos plaquetarios y de la coagulación. Los criterios de Paris planteados para esta entidad pueden tener una especificidad alta pero una sensibilidad alrededor del $20 \%$; por ello, se plantea que es recomendable considerar esta entidad cuando se presenta un paciente con criterios de HAI que cursa con colestasis o con criterios de CBP con hipertransaminasemia, asociado a la respuesta terapéutica. ${ }^{5}$ Esto fue lo que ocurrió en nuestra paciente.

La infección por SARS-CoV-2 puede comprometer diferentes órganos y sistemas, siendo uno de ellos el sistema inmune, reflejándose en la formación de auto anticuerpos y enfermedades mediadas por estos. ${ }^{3}$ Se ha reportado el desarrollo de enfermedades hepáticas autoinmunes e inclusive el síndrome overlap de HAI y CBP a raíz de la infección por este virus, aun no estando claro el mecanismo de desarrollo de esta enfermedad. ${ }^{7}$ En nuestro caso, la paciente presentaba el antecedente de artritis reumatoide que, a pesar de estar controlada, constituye un factor de riesgo para el desarrollo de otro tipo de enfermedades autoinmunes. Al ser expuesta a este virus y desarrollar COVID-19, es probable la desregulación del sistema inmune que conlleve al compromiso hepático.

En nuestra paciente, no solo se presentó un síndrome overlap, además se evidenció una trombosis de la vena porta, compromiso asociado a la hipercoagulabilidad que se presenta durante o en el estado post COVID-19. En estudios recientes se ha demostrado que el $21 \%$ de pacientes con esta enfermedad presenta complicaciones tromboembólicas venosas, elevándose esta frecuencia hasta el $31 \%$ en pacientes hospitalizados en unidades de cuidados intensivos. ${ }^{8}$ Se ha descrito trombosis de la vena porta en pacientes con COVID-19, que se presenta como dolor abdominal o hemorragia digestiva, principalmente. ${ }^{9}$ En este caso, debe tomarse en cuenta el compromiso hepático como factor de riesgo para el desarrollo de trombosis de la vena porta, siendo esta complicación frecuente en pacientes con cirrosis hepática avanzada o malignidad. ${ }^{10}$

Finalmente, el estudio de elastografía hepática clasificado como F2, que excluye el diagnóstico de cirrosis hepática, nos permite inferir que las complicaciones detalladas previamente se desarrollaron de forma aguda. La trombosis venosa portal se desarrolló principalmente por la infección por SARS-CoV-2 más que por el compromiso hepático que no era crónicamente avanzado. El sangrado digestivo por debió desarrollarse por la presencia del trombo, sustentado en la severidad de las VVEE (grado 3) con un compromiso hepático que no puede ser catalogado de cirrosis hepática como se ha mencionado.

En conclusión, el compromiso hepático en un paciente con COVID-19 puede deberse no solo a la propia infección por el SARS-CoV-2, sino que podría estar en relación al desarrollo de enfermedades autoinmunes con daño hepático como la HAI, CBP o un overlap entre ellos, así como la presencia de trombosis de la vena porta. Debe sospecharse una entidad inmunológica cuando las manifestaciones de injuria hepática persisten y progresan a pesar de haberse resuelto el cuadro agudo de COVID-19. La trombosis de vena porta es una complicación a plantearse en episodios de dolor abdominal o desarrollo de hipertensión portal manifestándose como hemorragia digestiva variceal.

\section{REFERENCIAS BIBLIOGRÁFICAS}

I. WHO Coronavirus (COVID-19) Dashboard [Internet]. URL disponible en: https://covid I 9.who.int

2. Machhi J, Herskovitz J, Senan AM, Dutta D, Nath B, Oleynikov MD, et al. The Natural History, Pathobiology, and Clinical Manifestations of SARS-CoV-2 Infections. J Neuroimmune Pharmacol. 2020;1-28.

3. Halpert G, Shoenfeld Y. SARS-CoV-2, the autoimmune virus. Autoimmun Rev. 2020; I (12): 102695.

4. Aguilar-Nájera O,Velasco-Zamora JA, Torre A. Overlap syndromes of autoimmune hepatitis: diagnosis and treatment. Rev Gastroenterol México (Engl Ed.) abril de 2015;80(2):150-9.

5. To U, Silveira M. Overlap Syndrome of Autoimmune Hepatitis and Primary Biliary Cholangitis. Clin Liver Dis. 2018;22(3):603-I I.

6. Rahim MN, Miquel R, Heneghan MA. Approach to the patient with acute severe autoimmune hepatitis. JHEP Rep. 2020;2(6): I00 I49.

7. Singh B, Kaur P, Maroules M. Autoimmune hepatitis-primary biliary cholangitis overlap syndrome triggered by COVID-19. Eur J Case Rep Intern Med. 2021;8(2):002264.

8. Malas MB, Naazie IN, Elsayed N, Mathlouthi A, Marmor R, Clary B. Thromboembolism risk of COVID-19 is high and associated with a higher risk of mortality: A systematic review and meta-analysis. EClinicalMedicine. 2020;29-30: 100639.

9. Hassan W, Ramadan HK-A. COVID-19 as a novel etiology of portal vein thrombosis: change in the current management concepts. Infect Dis. I de febrero de 2021;53(2): 148-50.

10. Intagliata NM, Caldwell SH, Tripodi A. Diagnosis, development, and treatment of portal vein thrombosis in patients with and without cirrhosis. Gastroenterology. 2019;156(6):I582-I599.el.

\section{CORRESPONDENCIA:}

Luis Enrique Carrasco Lozano

luis.carrasco9@unmsm.edu.pe

Fecha de recepción: 04-||-202I.

Fecha de aceptación: 30-|I-202I.

Conflicto de interés: ninguno, según los autores.

Financiamiento: por los autores. 